\title{
Detection threshold for percutaneous electrical stimuli: asymmetry with respect to handedness
}

\author{
W G FRIEDLI, P FUHR, W WIGET \\ From the Department of Neurology, University Hospital, Basel, Switzerland
}

SUMMARY Sensory strength-duration curves were obtained using percutaneous true square-wave pulses ranging from $0 \cdot 1$ to $20.0 \mathrm{~ms}$ produced by an isolated constant current stimulator. In 119 healthy volunteers sensory thresholds were measured bilaterally by stimulating the distal phalange of the little finger. In order to examine the relationship of sensory threshold and handedness the latter was assessed by means of the Edinburgh Inventory. An asymmetry of sensory threshold was found for all the subjects and this was more pronounced with shorter stimuli. Of right-handers tested $73.5 \%$ had a lower threshold on the left side while $70.8 \%$ of left-handers had a lower threshold on the right side. Although threshold asymmetry is associated with handedness this is not necessarily due to cerebral laterilisation.

When evaluating the usefulness of sensory strengthduration curve for assessment of sensory deficit in peripheral neuropathy we found sensory thresholds to be asymmetric in both upper and lower extremities of normal subjects. ${ }^{1}$ Detection thresholds for percutaneous electrical stimuli were lower for the left compared with the right side of right-handed people. Although the difference in absolute values was small, this asymmetry was a frequent finding in our population of healthy subjects.

The aim of the present study was to examine more systematically this threshold asymmetry and its relationship to handedness. We are not aware of any clinical report concerning quantitative assessment of cutaneous sensation with respect to handedness. While complex instruments are required for quantification of cutaneous sensation with "adequate" stimuli ${ }^{2}$ detection threshold for electrical pulses provided by an isolated constant-current stimulator has been shown to represent a reproducible measure. ${ }^{13}$ Larger amplitudes of sensory nerve action potentials were found in the non-dominant hand of both right- and left-handed subjects. ${ }^{4-6}$ Hence, the asymmetry of both the amplitude of sensory nerve action potentials and detection threshold for percutaneous electrical stimuli might be due to an asymmetry of peripheral sensory innervation. Alter-

Address for reprint requests: Walter G Friedli, Neurologische Universitätsklinik, Kantonsspital, CH-4031 Basel, Switzerland.

Received 20 May 1986. Accepted 23 September 1986. natively, threshold asymmetry which refers to various parts of the body might be governed by structural or functional asymmetry in the central nervous system.

Methods

Silver-silverchloride surface electrodes of $8 \mathrm{~mm}$ diameter filled with electrode cream were taped about $3 \mathrm{~cm}$ apart over the volar surface of the little finger. The electrode placed over the distal phalange was connected to the anode of an isolated stimulation system consisting of an impulse generator (Stimulator "F", Toennies) and a constant current stimulator. The latter was capable of producing true squarewave stimuli over the range of pulse durations tested. The skin was cleaned with ether and alcohol. During the measurement skin temperature was kept constant at $34^{\circ} \mathrm{C}$ by means of a temperature control unit (DISA, Type $15 \mathrm{H} \mathrm{02}$ ). Electrical stimuli were given at a rate of $0.5 \mathrm{~Hz}$. The amount of current in milliamperes required to produce a minimally perceptible sensation was measured for four different durations of square-wave pulses $(0 \cdot 10,0 \cdot 50,5 \cdot 0,20.0 \mathrm{~ms})$. Detection thresholds were obtained by a method of descending limits, continuously decreasing from a level at which the subject had a minimal sensation. Although not optimal from a scientific point of view ${ }^{7}$ the method of limits is reliable and easy to use in subjects who are properly prepared even if they are inexperienced in sensory testing. At least three independent measurements were performed on each subject for each pulse duration tested. The series started with stimuli of $20.0 \mathrm{~ms}$ duration, followed by $0 \cdot 10$, 5.0 and $0.50 \mathrm{~ms}$ stimuli. Measurements were performed on both sides in a random order and without regard to the subject's handedness. By plotting detection thresholds against stimulus duration separate sensory strength-duration curves were obtained for the right and the left hand. Threshold 


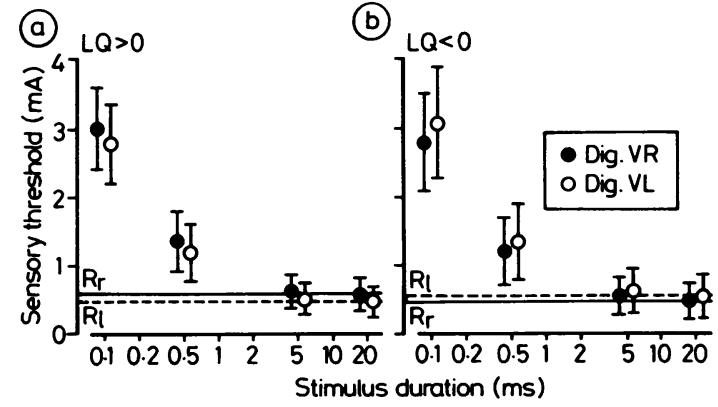

Fig 1 Strength-duration curves for rectangular current pulses on the volar surface of the little finger. (A) Average of 69 right handed subjects $(L Q>0)$ ranging in age from 10 to $71 \mathrm{yr}$ (median 35.0). (B) Average of 48 left handed subjects $(L Q<0)$ ranging in age from 18 to $65 \mathrm{yr}$ (median 29.5). Thresholds are separated for stimulation on the right (O) and on the left $(O)$ side in both groups. Rheobases are indicated for stimuli on the right $\left(R_{\mathrm{r}}\right)$ and on the left $\left(R_{1}\right)$ hand.

asymmetry was defined as the difference between the sensory threshold of the subject's right and left hand. While the magnitude was given in milliamperes, the direction of asymmetry was specified by a positive or negative sign according to whether the value was higher on the right or on the left side respectively. To ascertain the reproducibility of both absolute value and asymmetry of the sensory threshold one of the authors was tested in 15 independent series of measurements on different days.

Studies were performed on 119 healthy volunteers, 57 men

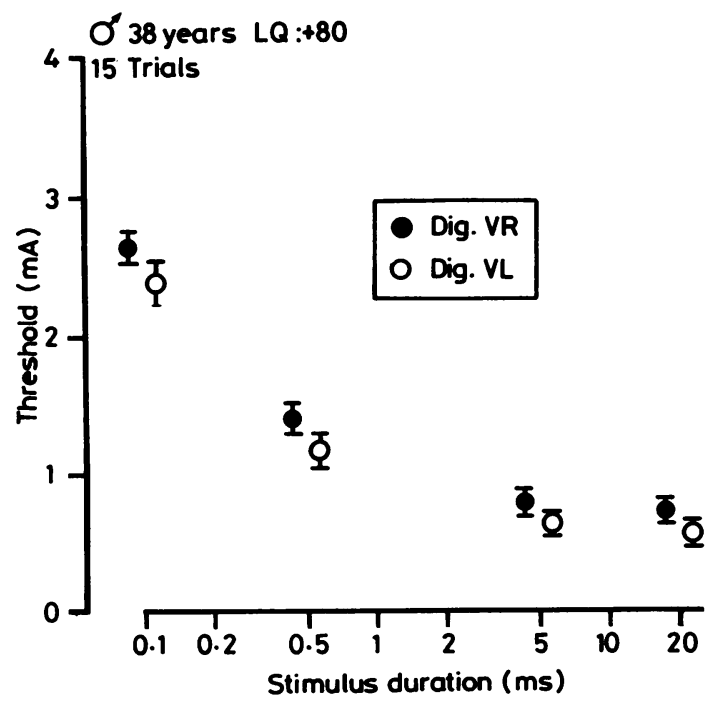

Fig 2 Reproducibility of both sensory threshold and threshold asymmetry for one right handed male subject. Average of 15 series of independent measurements performed on different days.

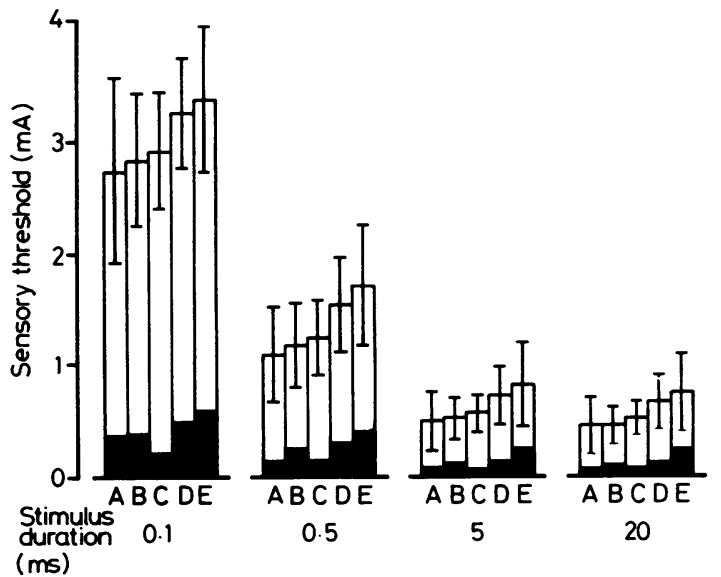

Fig 3 Sensory threshold in different age groups for the stimulus durations tested. Average values of absolute threshold for both hands (white columns) and absolute threshold asymmetry (black columns) without regard to handedness. $A: n=24,16-25 y r$ (median 23.0), $B: n=38$, 26-35 yr, (median 30.0), $C: n=13,36-45 \mathrm{yr}$ (median 41.5), $D: n=13,46-55 \mathrm{yr}($ median 49.0), $E: n=17,56-65 \mathrm{yr}$ (median 58.0).

and 62 women, ranging in age from 10 to 71 years (median 32.5 years), without history or clinical evidence of neuropathy. Handedness was assessed by means of the 10-item Edinburgh Inventory ${ }^{8}$ providing a "Laterality quotient" (LQ) for each subject tested. According to the sign of the LQ our population was composed of 69 right and 48 left handed subjects. The two groups were fairly comparable as to the absolute values of the $L Q$ : scores lay between +75 and +100 in $59 \%$ of our right handed population and ranged from -75 to -100 in $55 \%$ of left handed subjects. The LQ was zero in two volunteers.

The Edinburgh index was compared with both magnitude and direction of threshold asymmetry for percutaneous electrical stimuli. Various statistical procedures were used to seek possible correlations between LQ and asymmetry of sensory threshold.

\section{Results}

Detection thresholds for percutaneous electrical stimuli were closely reproducible in our subjects on successive trials. Although the task was subjectively more difficult with stimuli of $0.1 \mathrm{~ms}$, repetitive assessment of sensory threshold revealed consistent values also for these short electrical pulses. The characteristics of sensory strength-duration curves obtained in these experiments did match our former results. ${ }^{1}$ Threshold current decreased markedly with increasing stimulus durations up to $0.5 \mathrm{~ms}$ and less with longer stimuli (fig 1). The amount of current of infinite duration required to produce minimal sensation (rheobase) was achieved by pulse durations of $20 \mathrm{~ms}$. Reproducibility of the strength-duration curve for the 

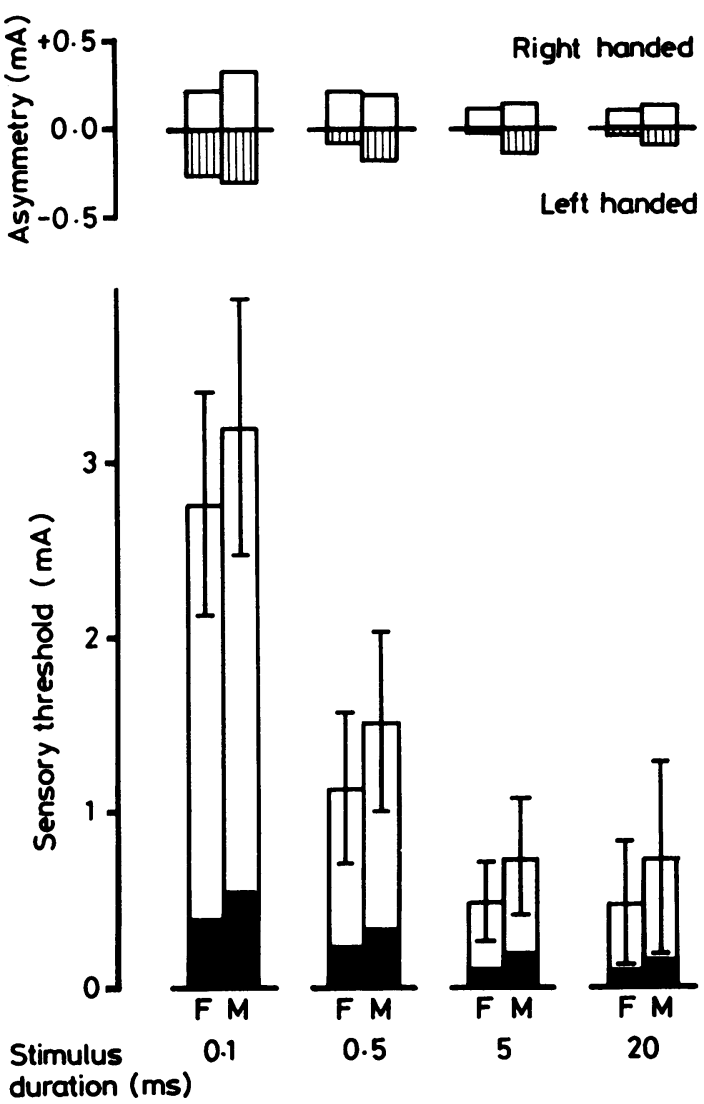

Fig 4 Sensory threshold in age-matched male and female subjects for various pulse durations. M: 40 males, 19-70 yr (median 30.0); $F$ : 40 females, 18-69yr (median 29.5). (A) Average threshold (white columns) and threshold asymmetry (black columns) in absolute values without regard to handedness. (B) Magnitude and direction of threshold asymmetry in consideration of handedness for the same groups. Average values for right (white columns) and left handed (hatched columns) subjects. Note opposite direction of asymmetry for subjects with positive ( 24 in $M, 22$ in F) and negative (16 in $M, 18$ in $F$ ) $L Q$.

same subject was demonstrated by 15 independent measurements on different days (fig 2).

Comparison of different age groups revealed an increase of absolute threshold values with increasing age for all stimulus durations tested which was more pronounced with shorter pulses (fig 3). Average threshold of 40 male subjects was compared with that of an age-matched group of 40 females without regard to handedness demonstrating lower values for the female group (fig 4). This sex-linked difference of absolute threshold was a consistent finding and of similar magnitude for the various pulse durations tested.
Asymmetry of sensory threshold was found in most of the subjects participating in these experiments. Both magnitude and direction of threshold asymmetry were positively correlated with handedness as indicated by the sign of the Edinburgh index. Although the absolute thresholds were of comparable size the direction of asymmetry was opposite for right and left handed subjects (fig 1A, B). The latter showed higher average thresholds on the left side while higher threshold current was found on the right side of right handers. There was a constant direction of asymmetry within the same subject also in a series of independent measurements as represented by small standard deviations of threshold values for either side which hardly overlapped each other (fig 2). However, the analysis of individuals did not provide a uniform behaviour of right and left handed subjects as to the direction of threshold asymmetry. Although the latter was related to handedness in the sense described above in most of right and left handed subjects, it was the reverse in a minority of them. This was true also for some strong right and left handed individuals and did not seem to correlate with the absolute value of the LQ. By plotting the LQ against threshold asymmetry for the whole population the relationship between the two parameters was visualised (fig 5 for $0.1 \mathrm{~ms}$-stimuli). Correlation between handedness and threshold asymmetry was given by Spearman rank correlation coefficients of 0.3655 for $0.1 \mathrm{~ms}$ stimuli, 0.3645 for $0.5 \mathrm{~ms}, 0.3791$ for $5.0 \mathrm{~ms}$ and 0.3891 for $20.0 \mathrm{~ms}$ stimuli (correlation coefficients for all stimulus durations are significantly different from $0, p<$ $0 \cdot 001$ ). For all stimulus durations tested the Wilcoxon rank sum test provided statistically significant evidence that the right- and left-handed population differed with respect to their threshold asymmetry ( $p$ $<0.001$ ). Parameters of threshold asymmetry are summarised in fig 6 . A positive asymmetry was found in $73.5 \%$ of right handed subjects for all pulse durations tested while the percentage of lefthanders with negative values varied from 70.8 for short stimuli to 62.5 and 56.3 for $5.0 \mathrm{~ms}$ and $20.0 \mathrm{~ms}$ pulses respectively. With longer stimuli there was not only a diminution of average threshold asymmetry in milliamperes but also an increasing number of subjects without any asymmetry at all. No asymmetry of sensory threshold was seen in three subjects $(2 \cdot 5 \%)$ with $0.1 \mathrm{~ms}$ stimuli while it was lacking in 14 subjects $(11.8 \%)$ with $20.0 \mathrm{~ms}$ pulses. Interestingly, both right and left handed subjects without asymmetry with longer stimuli were found mainly in the group showing a negative asymmetry with decreasing stimulus duration.

Generally, threshold asymmetry was more pronounced with shorter stimuli and seemed to be proportionally related to the magnitude of absolute 


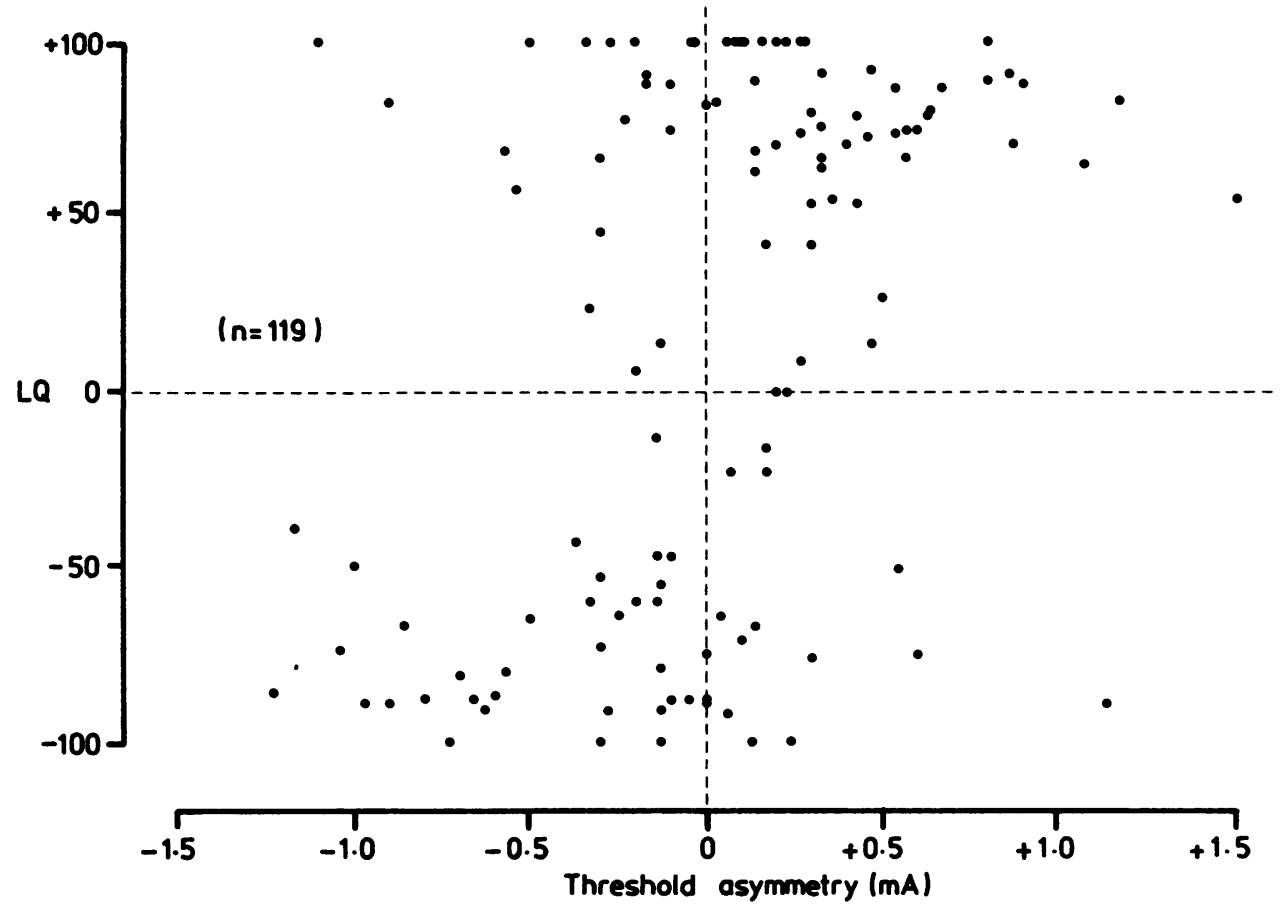

Fig 5 Relationship between laterality quotient ( $L Q)$ and threshold asymmetry for electrical pulses of $0 \cdot 1 \mathrm{~ms}$. Positive sign of asymmetry indicates a higher threshold on the right compared with the left side. Right handers are defined by a positive $L Q(n=69)$, left handers by a negative $L Q(n=48)$. See text for further details.

(a)

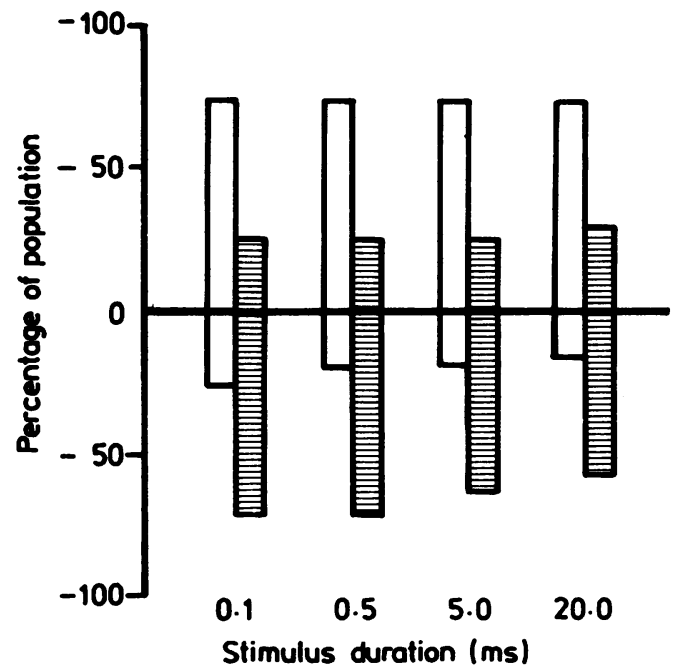

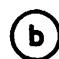

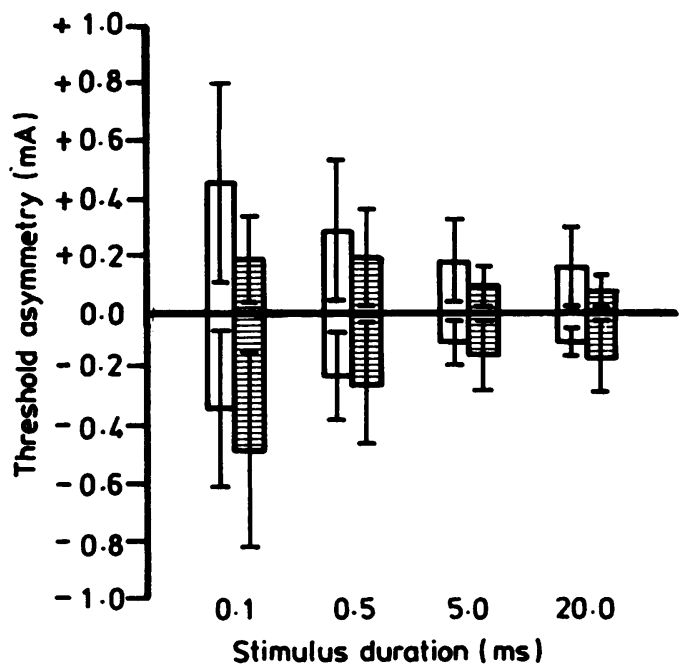

Fig 6 Parameters of threshold asymmetry in right (white columns) and left handed (hatched columns) subjects for various pulse durations. (A) Percentages of the right $(69=100 \%)$ and left handed $(48=100 \%)$ population with positive or negative sign of threshold asymmetry. (B) Average threshold asymmetry in $m A$ (mean $\pm 1 S D$ ) in positive or negative direction for right and left handers corresponding to the groups in Fig A. See text for further details. 
thresholds (figs 1, 2). Correspondingly, the asymmetry of sensory threshold in milliamperes showed an increasing tendency with growing age (fig 3). Although fig 4 suggests lower values of threshold asymmetry in females than in males, this could not be supported by comparing groups consisting of 20 right handed subjects each and matched with respect to both degree of handedness (LQ > 75) and age (onetailed $t$ test for paired observations not significant at the 0.05 level). Statistical power ${ }^{9}$ was about $89 \%$ with $5.0 \mathrm{~ms}$ stimuli for a difference of at least $0.1 \mathrm{~mA}$ between the two means, whereas it was around $33 \%$ for shorter stimuli and about $66 \%$ for $20.0 \mathrm{~ms}$ pulses. These results allow with reasonable probability the conclusion that in reality there exists no relevant sex difference with respect to threshold asymmetry at least with $5.0 \mathrm{~ms}$ stimuli.

\section{Discussion}

As in our previous observations ${ }^{1}$ the present data demonstrate the asymmetry of sensory threshold for percutaneous electrical stimuli. Our results demonstrate a clear relationship between handedness and threshold asymmetry. Among all 69 right handed subjects tested $50(73.5 \%)$ show a lower threshold on the left hand while in 34 of the $48(70.8 \%)$ left handers tested less current is required for perception of short electrical pulses on the right side.

A superiority of the left side of the body for pressure sensitivity and two-point discrimination has been found on the extremities of right handed men and women. ${ }^{10}$ However, we are not aware of any clinical report concerning quantitative assessment of cutaneous sensation with adequate stimuli with respect to handedness in healthy subjects. ${ }^{11-15}$ Although studied by various electrodiagnostic techniques ${ }^{16-19}$ excitability of peripheral nerves has been investigated without regard to handedness. The question arises as to the basis of the present findings. A number of factors other than cerebral hemispheric asymmetry of function could contribute to this lateral asymmetry. The fact that threshold asymmetry was found in various parts of the body (for example fig 1 of reference 1 ) rules out an asymmetry on the basis of minor mechanical damage to the nerve ${ }^{20}$ which was likely to be more marked in the dominant hand. Further insight into the problem requires some remarks on the nature of percutaneous electrical stimuli. The nerve action potentials to electrical pulses have been shown to be similar in shape to those evoked by adequate stimuli applied to the digits. ${ }^{21}$ The amplitude of the sensory nerve action potential represents the number of nerve fibres activated by electrical stimuli. ${ }^{22}$ There is a strong resemblance of the behavioural strengthduration curves ${ }^{13}$ to those obtained in electro- physiologic studies, ${ }^{16}$ that is, psychophysical functions resemble those obtained by measuring currents required to activate nerve fibres at different stimulus durations. ${ }^{22}$ Electric pulses bypass the receptors and directly initiate the action potential at the peripheral nerve ${ }^{23-26}$ whereas the sequence begins at the receptor for tactile stimuli. ${ }^{27}$ Hence, since the sensory threshold is proportionally related to the number of nerve fibres activated, its asymmetry may be contingent on an asymmetry of sensory innervation in the periphery (assuming that single nerve fibres show equal electrical excitability on both sides). Larger amplitudes of sensory nerve action potentials have been described in the non-dominant hand of both right handed adults ${ }^{4}$ and children ${ }^{5}$ while this asymmetry was reverse in left handed subjects. ${ }^{6}$ Although not based upon an analysis of intersubject consistency of the laterality difference these findings suggest some dependence of sensory innervation on handedness. Moreover, the increase of sensory threshold with growing age (fig 3) is compatible with factors concerning the peripheral nerve. Alterations in amplitude, duration and latency of the nerve action potential have been observed as age-dependent features of the peripheral sensory nerve ${ }^{28}$ whereas the mean central conduction velocity from cervical spinal cord to post-central cortex was found to remain constant with aging. ${ }^{29}$ Changes in the peripheral nerve can be related both to mild segmental demyelination and to some loss of nerve fibres ${ }^{30}$ while histometric studies have revealed a decreasing fibre density with? increasing age. ${ }^{31} 32$ On the other hand, a decrease of numbers of total and large myelinated fibres with age has been found also in the posterior column of the spinal cord. ${ }^{33}$

Since the central somatosensory pathway crosses the midline in the medulla one can infer that the lower left side threshold in right handed subjects reflects greater right hemisphere involvement in perception of the stimuli presented here. The possible dependence of sensory threshold asymmetry to cerebral hemispheric asymmetry of function is an attractive idea. Based on clinical observations ${ }^{34-36}$ the right hemisphere has been recognised as dominant for certain nonverbal functions, particularly the analysis of external space and the orientation of the body within this space. According to the fact that neglect is seen more frequently with right hemisphere lesions ${ }^{37}$ the right hemisphere is also of predominant importance in mediating attention. However, left handed subjects did show a reversed pattern, lower thresholds on their non-dominant right side. Certain aspects of right hemisphere dominance are thought to be conservative, including only rare shifting to the left hemisphere even in people without strong left-hemisphere dominance for language and handedness. ${ }^{38}$ Contrary 
to our results one would expect therefore a smaller number of left-handers to show lower thresholds on the right side if there was a relationship between threshold asymmetry and functions dominated by the right hemisphere. On the other hand, the observed distribution of threshold asymmetry with a reversed but otherwise similar pattern for right and left handed subjects is rather unlikely to correspond with language dominance, since the latter is clearly less associated with handedness than the asymmetry of sensory threshold observed here. The absence of a reliable difference between adult right handed males and females in threshold asymmetry also suggests that some factor other than cerebral dominance might be responsible for asymmetry of sensory threshold, since there is evidence for the female brain to have more bilateral cerebral organisation both for verbal and nonverbal functions. ${ }^{39}$

Based on these, theoretical considerations one should be cautious in interpreting the asymmetry of sensory threshold as reflecting an aspect of cerebral lateralisation. ${ }^{40}$ Electrophysiological data $^{41}$ which will be the subject of a subsequent paper rather indicate a peripheral origin of sensory threshold asymmetry. We feel, that it is important to be aware of this asymmetry when assessing sensory innervation of restricted skin areas not only by measuring sensory thresholds ${ }^{1}$ but also by using somatosensory evoked potentials to near-threshold stimuli. ${ }^{42}$

A preliminary account of some of the findings was presented at XIth International Congress of Electroencephalography and Clinical Neurophysiology, London, August 1985.

The authors are indebted to Mr K Hediger for technical support. We are also grateful to Dr M Hallett for his helpful criticism of the manuscript and to $\mathrm{Dr}$ M Oberholzer for statistical considerations.

\section{References}

1 Friedli WG, Meyer M. Strength-duration curve: a measure for assessing sensory deficit in peripheral neuropathy. J Neurol Neurosurg Psychiatry 1984;47:184-9.

2 Dyck PJ, Zimmermann IR, O'Brien PC, et al. Introduction of automated systems to evaluate touchpressure, vibration, and thermal cutaneous sensation in man. Ann Neurol 1978;4:502-10.

3 Rollman GB. Behavioral assessment of peripheral nerve function. Neurology 1975;25:339-42.

4 Cruz Martinez A, Barrio M, Pérez Conde MC, Ferrer MT. Electrophysiological aspects of sensory conduction velocity in healthy adults. 2 . Ratio between the amplitude of sensory evoked potentials at the wrist on stimulating different fingers in both hands. J Neurol Neurosurg Psychiatry 1978;41:1097-101.

5 Cruz Martinez A, Pérez Conde MC, Del Campo F, Bar- rio M, Gutiérrez AM, Ferrer MT. Sensory and mixed conduction velocity in infancy and childhood. 1 . Normal parameters in median, ulnar and sural nerves. Electromyogr Clin Neurophysiol 1978;18:487-504.

6 Cruz Martinez A, Pérez Conde MC, Del Campo F, Mingo P, Ferrer MT. Ratio between the amplitude of sensory evoked potentials at the wrist in both hands of left-handed subjects. J Neurol Neurosurg Psychiatry 1980;43:182-4.

7 Gescheider GA. Psychophysics. Method and theory. Chichester: John Wiley and Sons, 1976.

8 Oldfield RC. The assessment and analysis of handedness: the Edinburgh Inventory. Neuropsychologia 1971;9: 97-115.

9 Cohen J. Statistical Power Analysis for the Behavioral Sciences. New York: Academic Press, 1977.

10 Weinstein S. Intensive and extensive aspects of tactile sensitivity as a function of body part, sex and laterality. In: Kenshalo D, ed. The Skin Senses. Springfield, Illinois: CC Thomas, 1968:195-222.

11 Lindblom U. Quantitative testing of sensibility including pain (chap 8). In: Stalberg E, Young RR, eds. Neurology, Vol 1: Clin Neurophysiology. London: Butterworth, 1981:167-90.

12 Fruhstorfer H, Lindblom U, Schmidt WG. Method for quantitative estimation of thermal thresholds in patients. JNeurol Neurosurg Psychiatry 1976;39: 1071-5.

13 Goldberg JM, Lindblom U. Standardised method of determining vibratory perception thresholds for diagnosis and screening in neurological investigation. J Neurol Neurosurg Psychiatry 1979;42:793-803.

14 Lynn A, Perl ER. A comparison of four tests for assessing the pain sensitivity of different subjects and test areas. Pain 1977;3:353-65.

15 Dyck PJ, O'Brien PC, Bushek W, Oviatt KF, Schilling $\mathrm{K}$, Stevens JC. Clinical vs quantitative evaluation of cutaneous sensation. Arch Neurol 1976;33:651-5.

16 Heckmann JR. Excitability curve: a new technique for assessing human peripheral nerve excitability in vivo. Neurology 1972;22:224-30.

17 Powers RE, Swick HM, McQuillen MP. Human nerve excitability. JNeurol Neurosurg Psychiatry 1978; 41:642-8.

18 Wright EA, McQuillen MP. Hypoexcitability of ulnar nerve in patients with normal motor nerve conduction velocities. Neurology 1973;23:78-83.

19 Veale JL, Mark RF, Rees S. Differential sensitivity of motor and sensory fibers in human ulnar nerve. J Neurol Neurosurg Psychiatry 1973;36:75-86.

20 Neary D, Ochoa J, Gilliatt RW. Sub-clinical entrapment neuropathy in man. $J$ Neurol Sci 1975;24:283-98.

21 McLeod JG. Digital nerve conduction in the carpal tunnel syndrome after mechanical stimulation of the finger. J Neurol Neurosurg Psychiatry 1966;29:12-22.

22 Buchthal F, Rosenfalck A. Evoked action potentials and conduction velocity in human sensory nerves. Brain Res 1966;3:1-122.

23 Rollman GB. Electrocutaneous stimulation: Psychometric functions and temporal integration. Percept Psychophys 1969;5:289-93.

24 Rollman GB. Detection models: Experimental tests with 
electrocutaneous stimuli. Percept Psychophys 1969;5:377-80.

25 Hawkes GR. Cutaneous discrimination of electrical intensity. Am J Psychol 1961;74:45-53.

26 Higgins JD, Tursky B, Schwartz GE. Shock-elicited pain and its reduction by concurrent tactile stimulation. Science 1971;172:866-7.

27 Rollman GB. Tactile stimulation: psychophysical studies of receptor function. Percept Psychophys 1973;13: 81-6.

28 Buchthal FA, Rosenfalck A, Behse F. Sensory potentials of normal and diseased nerve. In: Dyck PJ, Thomas PK, Lambert EH, Bunge R, eds. Peripheral Neuropathy Vol. 1. Philadelphia: Saunders, 1984:981-1015.

29 Desmedt JE, Cheron G. Somatosensory evoked potentials to finger stimulation in healthy octogenarians and in young adults: wave forms, scalp topography and transit times of parietal and frontal components. Electroencephalogr Clin Neurophysiol 1980;50:404-25.

30 Lascelles RG, Thomas PK. Changes due to age in internodal length in the sural nerve in man. J Neurol Neurosurg Psychiatry 1966;29:40-4.

31 O'Sullivan DJ, Swallow M. The fibre size and content of the radial and sural nerves. JNeurol Neurosurg Psychiatry 1968;31:464-70.

32 Tackmann W, Spalke G, Oginszus HJ. Quantitative histometric studies and relation of number and diameter of myelinated nerve fibers to electrophysiological parameters in normal sensory nerves of man. $J$ Neurol
(Berlin) 1976;212:71-84.

33 Ohnishi A, O'Brien PC, Okazaki H, Dyck PJ. Morphometry of myelinated fibers of fasciculus gracilis of man. J Neurol Sci 1976;27:163-72.

34 Ettlinger G, Warrington E, Zangwill OL. A further study of visuo-spatial agnosia. Brain 1957;80:335-61.

35 Semmes J, Weinstein S, Ghent L, Teuber HL. Correlates of impaired orientation in personal and extrapersonal space. Brain 1963;88:747-72.

36 Milner B, Taylor L. Right hemisphere superiority in tactile pattern recognition after cerebral commissurotomy: Evidence for non-verbal memory. Neuropsychologia 1972;10:1-15.

37 Critchley M. The Parietal Lobes. London: Edward Arnold Comp, 1953.

38 Geschwind N, Galaburda AM. Cerebral lateralization. Biological mechanisms, associations and pathology: I. A hypothesis and a program for research. Arch Neurol 1985;42:428-59.

39 McGlone J. Sex differences in human brain asymmetry: a critical survey. Behav Brain Sci 1980;3:215-63.

40 Colburn CJ. Can laterality be measured? Neuropsychologia 1978;16:283-9.

41 Friedli WG, Wiget W, Kaeser HE. Detection threshold for electrical stimuli-a laterality measure? Electroencephalogr Clin Neurophysiol 1985;61:152-3.

42 Desmedt JE. Nouvelles méthodes d'objectivation des dommages corporels chez l'homme. Bull Acad Natn Méd (Paris) 1977;161:88-91. 